\title{
Clear Aligners: An Update
}

Shahindah Shah*

Maharishi Markandeshwar College of Dental Sciences and Research, Mullana, Ambala, Haryana, India

$\begin{array}{cl}\text { Article History } & \text { Abstract: Clear aligners have revolutionized the field of esthetic orthodontics and the } \\ \text { number of patients keen to get clear aligner therapy is increasing with time. To meet } \\ \text { Received: } 17.11 .2020 & \text { this demand, the clear aligner technology is becoming more versatile day by day. This } \\ \text { Accepted: } 27.11 .2020 & \text { paper will throw some light on clear aligner therapy highlighting the clinical scope, } \\ \text { Published: } 02.12 .2020 & \text { case selection and treatment. } \\ & \text { Keywords: Revolutionized clinical scope aligners esthetic orthodontics. }\end{array}$

https://www.easpublisher.com

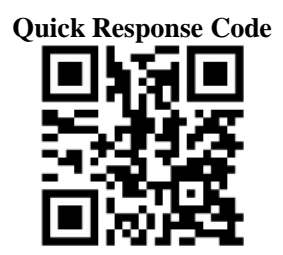

Copyright () 2020 The Author(s): This is an open-access article distributed under the terms of the Creative Commons Attribution 4.0 International License (CC BY-NC 4.0) which permits unrestricted use, distribution, and reproduction in any medium for non-commercial use provided the original author and source are credited.

\section{INTRODUCTION}

The demand for esthetic orthodontic appliances has increased in recent years [1]. Many appliances like lingual orthodontics, ceramic/composite brackets and clear plastic appliances have been developed to address this demand. The most remarkable advancement in the last decade has been the introduction of clear aligners. Clear aligners are series of thermoformed appliances fabricated from transparent thermoplastic material. Each aligner is worn for about a 2 week period and brings about $0.25-0.3 \mathrm{~mm}$ of tooth movement. Patient compliance is very important for good results since the appliance should be worn for about 20-22 hours in a day [2].

\section{HISTORY}

1945: Kesling introduced tooth positioners [3].

1964: Dr Henry Nahoum introduced the vaccum formed dental contour appliance [4].

1971: Pontiz gave the "invisible retainer" - a thermoformed plastic appliance. Mc Namara and others also used such retainers for minor orthodontic tooth movement [5].

1993: Sheridan developed Essix appliance for long term retention [6]. Hilliard and Sheridan further developed Essix appliance introducing a series of thermoforming pliers [7].

1998: Invisalign, the pioneer in clear aligners was established. Since then innumerable clear aligner companies have been set up for example ClearCorrect, OrthoClear, NovoAlign etc

\section{Case selection for clear aligners}

Gabriele Rossini et al. [8] concluded in their study that clear aligners can be used for leveling and alignment in non-growing subjects, anterior intrusion and molar bodily movement. Anterior extrusion and rotations of round teeth cannot be effectively achieved by clear aligner therapy.

Joffe[9] has given the following criteria for case selection for clear aligner therapy:

1. Mild crowding (1-5m)

2. Mild spacing $(1-5 \mathrm{~mm})$

3. Deep bite

4. Narrow arches

The aspects difficult to handle with clear aligners according to Joffe ${ }^{9}$ are as follows:

1. Over $5 \mathrm{~mm}$ of spacing or crowding.

2. More than of $2 \mathrm{~mm}$ of sagittal discrepancies.

3. Large discrepancy between centric occlusion and centric relation.

4. More than 20 degrees of rotations and more than 45 degrees of tipping.

5. Open bite and extrusion.

6. Short clinical crowns and multiple missing teeth. 


\section{Digitalized treatment planning}

Once the case is selected, the records including impressions, radiographs, intraoral and extraoral photographs are submitted to the manufacturing company. The impressions and initial bite can either be submitted in physical form or in the form of 3D scanned images. Once the records are submitted, the clinician needs to fill a prescription form on the company's website. This form reflects the treatment goals based on the clinician's experience and knowledge. The prescription goes through a list of objectives step by step. This is followed by re-positioning the teeth virtually on the computer using special software for e.g, Treat software.

\section{Visualizing the treatment plan}

A remarkable attribute of clear aligners is that the treatment alternatives can be visualized by the clinician and then he can choose the best one. There are special softwares like 'Clincheck' for the visualization of the treatment plan submitted via the prescription form.

\section{Staging [10]}

Staging is the sequence in which and the speed at which the teeth are moved with aligners. Once the initial and the desired final position of the teeth are decided, corrective paths are devised between the two. Multiple clear aligners are then manufactured to move the teeth, to the various positions along the corrective path. Staging is of following types:

Segmented staging: In segmented staging, one small group of teeth is moved and another group is kept stationary which provides anchorage.

Simultaneous staging: It is also known as Xstaging. All the teeth of an arch are moved together from initial to the final stage.

$V$-staging: This is for the maxillary arches. It starts with the distalization of maxillary molars, followed by bicuspids, and ends with retraction of anterior teeth.

A-staging: This is the opposite of $\mathrm{V}$-staging and can be used in both the maxillary and mandibular arches. It is characterized by movement of the anterior teeth in the anterior direction, followed by the posterior teeth.

$M$-staging: This is used for premolar extraction cases. This staging pattern commences by closing the extraction spaces followed by leveling and alignment.

\section{Manufacture}

Clear aligners are manufactured by the rapid prototyping technology called stereolithography. The gist of this technology is based on breaking down three dimensional computer models into thin cross sectional layers, followed by physical generation of these three dimensional objects layer by layer. After their fabrication, the aligners are trimmed and polished. The patient's initials, case number, aligner number, and arch (upper or lower) are laser etched onto the aligners after which they are disinfected, packed and shipped to the doctor's office.

\section{Clear aligner material}

Clear aligners are made of polyurethane which is a clear thermoplastic material. This is the material of choice because of its unique properties of flexibility, transparency, breakage resistance and ability to exert light forces. Some manufacturing companies use polyethylene terephthalate glycol-modified (PET-G), polypropelene, polycarbonate (PC), ethylene vinylacetate, Zendura (rigid polyurethane) among many others. In 2013, SmartTrack was released by Invisalign which is a multilayered polyethylene and copolyester material.

\section{Attachments, power ridges and auxillaries [10, 11]}

The clear aligners can now be used to bring about complex tooth movements using attachments, power ridges and auxillaries.

\section{Attachments}

These are tiny composite additions to the tooth surface that enhance areas of undercuts either for retention or to facilitate particular movements.

Optimized Attachments: These are generated automatically by a sophisticated series of software tools. Their shapes are determined by computer codes or algorithms.

Manually Placed Attachments: These are chosen by the orthodontist himself from the choices available. Hence, they are also called physician prescribed attachments.

\section{Based on the shape, attachments are of following types \\ Ellipsoid: These attachments are $0.75-1 \mathrm{~mm}$} thick, $2 \mathrm{~mm}$ wide and $3 \mathrm{~mm}$ high. They can be used singly or in pairs. They bring about derotations when used singly (Figure 1). And they can upright teeth when used in pairs, in which case they produce moment of couple (Figure 2).

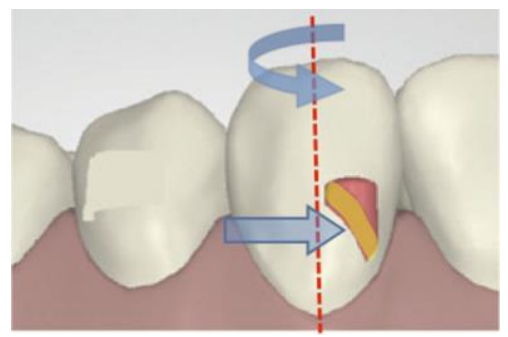

Fig-1: Schematic representations of single ellipsoid precision attachment used for derotating a canine 


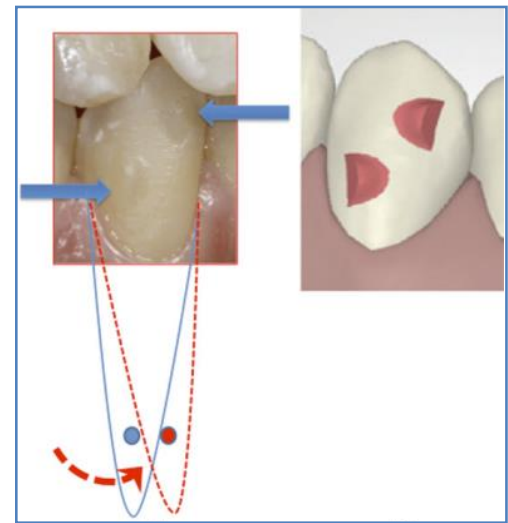

Fig-2: Ellipsoid precision attachments in a pair

Rectangular: These are $0.5-1 \mathrm{~mm}$ thick, $2 \mathrm{~mm}$ wide and 3,4 or $5 \mathrm{~mm}$ high. The rectangular attachments bring about bodily movement of teeth (Figure 3).

Bevelled: These are 0.25 to $1.25 \mathrm{~mm}$ thick, 3,4 or $5 \mathrm{~mm}$ wide and $2 \mathrm{~mm}$ high. They are most commonly used to extrude a tooth (Figure 4).

When initially bonded to the tooth surface, attachments are not fully engaged. This is called 'preactivation'. The attachments become more active as the patient graduates through successive aligners, until the aligner slot is fully filled. In order to allow unimpeded tooth movement in the desirable direction, some aligners have excess space opposite the active surface.

POWER RIDGES: They can be defined as corrugations present near the gingival margin of teeth (Figure 5). These facilitate control of torque. They have two main functions:

1. They add resiliency to the gingival third of the margin by making it stiffer.

2. They increase the moment arm of the aligner by providing additional force close to the gingival margin.

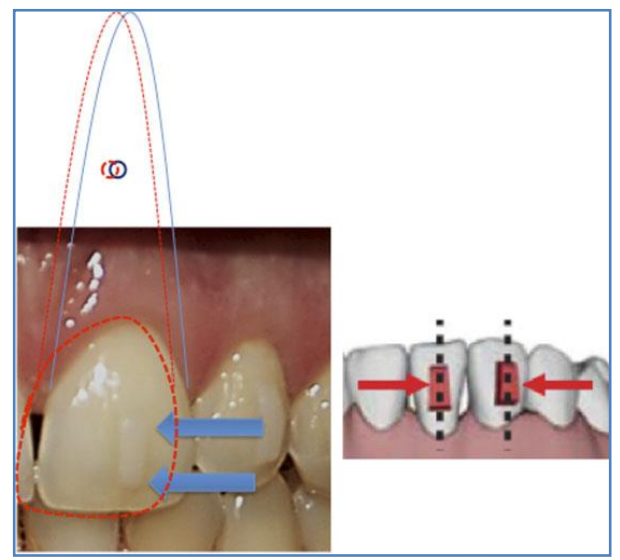

Fig-3: Rectangular attachments

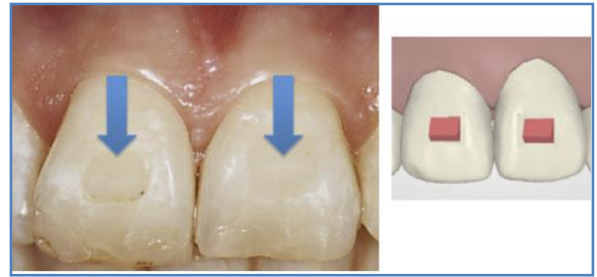

Fig-4: Bevelled Attachments

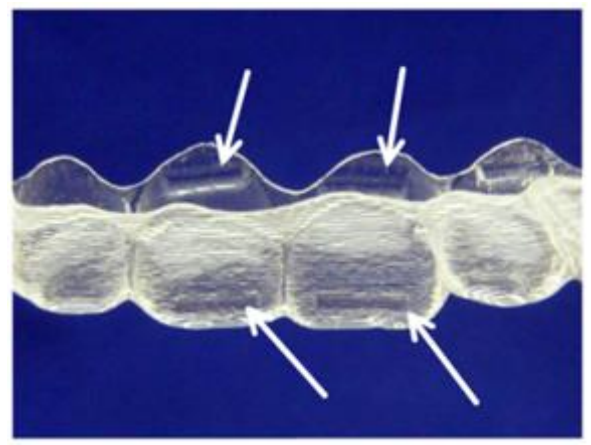

Fig-5: Power Ridges

\section{AUXILLARIES}

The auxillaries used with the aligners are elastics and miniscrews (Figures 6 \& 7). Special precision hooks, button cut-outs or power arms are incorporated into the aligners to attach the elastics. Miniscrews can also be used in a similar way as in fixed appliances, especially to bring about intrusion.
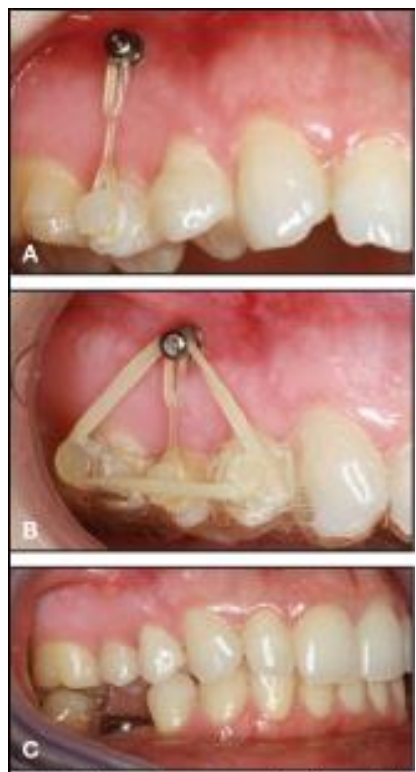

Fig-6: TADs in combination with aligners

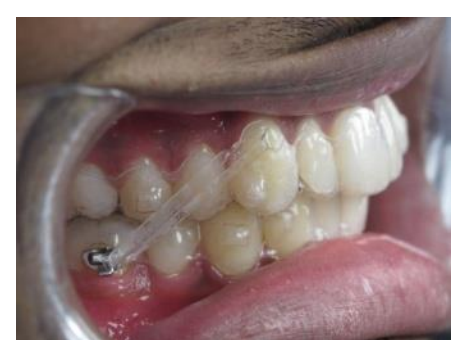

Fig-7: Elastics with precision hooks and button cut-outs 


\section{Biomechanics [10]}

When an aligner is worn, the undercuts of teeth provide retention whereas the active component is provided by the following two factors:

Elastic deformation: In this case, the entire body of the aligner gets distorted when it is worn. Slowly, with passage of time, it returns to its original shape carrying the tooth with it. However, the deformation must not be so great that it overpowers the retentive forces and leads to dislodgement of the aligners. To overcome this, the total desired tooth movement is divided into smaller stages.

Stretching of the matrix: In this case, the aligner stretches within the matrix of the plastic and then shrinks back to its original size carrying the tooth with it. This leads to the tooth movement in the vertical direction. These movements are also divided into small increments.

The moments and couples are created by the attachments and auxillaries.( Figures $8 \& 9$ ) In the force moment diagram we can see how moment is created with the help of an attachment on the incisal surface. A net force of $40 \mathrm{~g}$ (base level force of an aligner after 48 hours) intended to move the tooth lingually would require a moment of 320 to $400 \mathrm{~g}$-mm $(\mathrm{M} / \mathrm{F}$ ratio $8-10)$ for bodily movement or greater than $400 \mathrm{gmm}(\mathrm{M} / \mathrm{F}>10)$ for palatal root movement.

\section{Root control [10]}

It is a frequent observation in premolar extraction cases that the maxillary molars tend to tip mesially. This is reffered to as "dumping". To overcome this, the clear aligner technology has devised "Twin attachments" (Figure 10). Two $2 \mathrm{~mm}$ x $2 \mathrm{~mm} x$ $2 \mathrm{~mm}$ attachments are placed on the upper first and second molars. These create the desired couple to prevent the undesirable dumping.

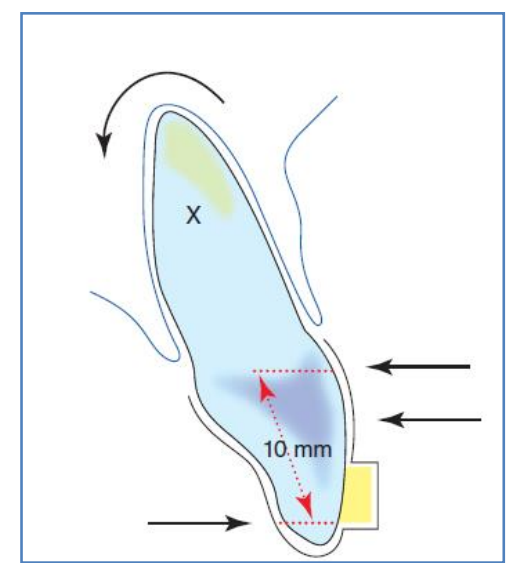

Fig-8: Force moment diagram with attachment on incisal surface

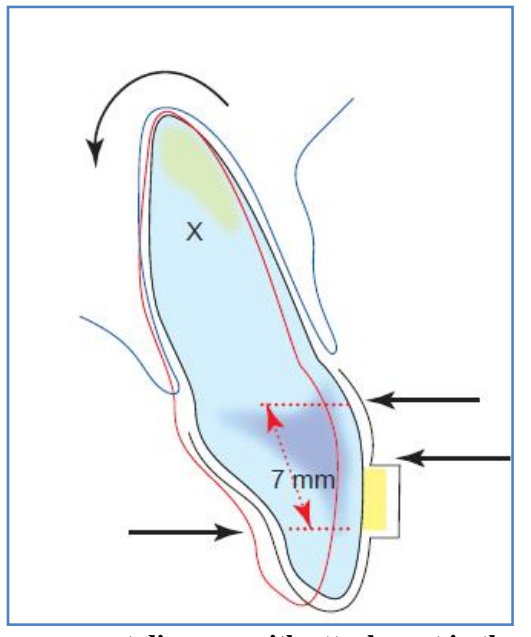

Fig-9: Force-moment diagram with attachment in the middle of the tooth

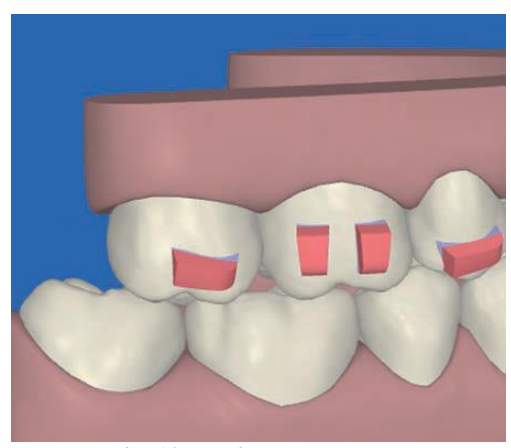

Fig-10: Twin attachments

\section{RETENTION}

A retention protocol similar to the fixed appliances is followed for clear aligners. The final aligner in the sequence or its thicker version is worn as a retainer for six months followed by night time wear. The various retainers which can be used after clear aligner therapy are:

1. Clear retainers also known as vacumm formed retainers.

2. Hawleys or Begg type retainer.

3. Fixed bonded retainer.

\section{Advantages of clear aligners}

In addition to their esthetic and hygienic benefits to the patients, the impressionless scanning performed, also subjects the patients to less discomfort. Digital visualization of the treatment too, is an exceptional advantage. Two more commendable advantages of clear aligners are:

Less Root resorption: It is seen that the clear aligners exert light forces which help in maintaining the blood circulation and promote the formation of osteoblast and osteoclast progenitors. This decreases the risk of hyalinization which is an important factor that leads to root resorption. Furthermore, the intermittent forces applied on the teeth by the aligners provide enough time for the cementum to heal as well as prevent further resorption $[12,13]$. Various studies [14, 
15] have been conducted that prove that the root resorption caused by aligners is less.

Better Periodontal Health- Due to its design, the patients are able to maintain good oral hygiene. They spend more time flossing and brushing their teeth. This leads to a healthier periodontium. Low et al and Levirin et al. conducted studies on the oral biofilms of patients undergoing treatment with fixed appliances and clear aligners. They found that the bacteria present in the clear aligner biofilm were associated to a lower risk of periodontal disease

\section{DISADVANTAGES}

Clear aligners are highly accepted because of esthetic reasons. However, they pose certain disadvantages:

1. They are unable to treat complex malocclusions.

2. The role of clinician becomes limited whereas greater role is played by the softwares and manufacturing companies.

3. Patient compliance is critical for clear aligner therapy.

4. Many allergic reactions like blisters, swollen lips, laryngospasm, etc. have been reported with clear aligners because of Isocyanate which is a major component required for the synthesis of polyurethane.

5. The treatment time is lengthened because of all the documentation that needs to be done.

\section{CONCLUSiON}

Clear aligner technology has made its mark in the field of orthodontics. Over the past 20 years, clear aligners have become capable of treating many complex cases using advancements in technology like intraoral scanners, softwares like Treat and Clincheck, CADCAM and stereolithography. Moreover aligner materials and attachments continue to evolve to provide a better fit and more efficient treatment outcomes.

\section{REFERENCES}

1. Aakash, M. S., Purvesh, M. S., \& Romil, B. S. (2017). Clear aligners in orthodontics. Guident, 10(6).

2. Phan, X., \& Ling, P. H. (2007). Clinical limitations of Invisalign. Journal of the Canadian Dental Association, 73(3).
3. Kesling, H. D. (1945). The philosophy of the tooth positioning appliance. American Journal of Orthodontics and Oral Surgery, 31(6), 297-304.

4. Nahoum, H. I. (1964). The vacuum formed dental contour appliance. NY State Dent J, 9, 385-390.

5. Ponitz, R. J. (1971). Invisible retainers. American journal of orthodontics, 59(3), 266-272.

6. JJ, S. (1993). Ledoux W, McMinn R. Essix retainers: fabrication and supervision for permanent retention. J Clin $O \sim$ hod, 27(1), 37-45.

7. Hilliard, K., \& Sheridan, J. J. (2000). Adjusting Essix Appliances at Chairside-These simple adjustments can be made in the operatory. Journal of Clinical Orthodontics, 34(4), 236-238.

8. Rossini, G., Parrini, S., Castroflorio, T., Deregibus, A., \& Debernardi, C. L. (2015). Efficacy of clear aligners in controlling orthodontic tooth movement: a systematic review. The Angle Orthodontist, 85(5), 881-889.

9. Joffe, L. (2003). Invisalign $\AA: \quad$ early experiences. Journal of orthodontics, 30(4), 348352.

10. Graber, V.V. (2012). Orthodontics - Current principles $\&$ techniques $-5^{\text {th }}$ ed: Elsevier - Mosby; 778-811

11. Hennessy, J., \& Al-Awadhi, E. A. (2016). Clear aligners generations and orthodontic tooth movement. Journal of orthodontics, 43(1), 68-76.

12. Reitan, K. (1964). Effects of force magnitude and direction of tooth movement on different alveolar bone types. The Angle Orthodontist,34(4), 244255.

13. Dougherty, H. L. (1968). The effect of mechanical forces upon the mandibular buccal segments during orthodontic treatment. American journal of orthodontics, 54(2), 83-103.

14. Aman, C., Azevedo, B., Bednar, E., Chandiramami, S., German, D., Nicholson, E., ... \& Scarfe, W. C. (2018). Apical root resorption during orthodontic treatment with clear aligners: A retrospective study using cone-beam computed tomography. American Journal of Orthodontics and Dentofacial Orthopedics, 153(6), 842-851.

15. Fowler, B. (2010). A comparison of root resorption between invisalign treatment and contemporary orthodontic treatment. University of Southern California. 